Palabra Clave (La Plata), octubre 2017, vol. 7, n 1, e030. ISSN 1853-9912

Universidad Nacional de La Plata.

Facultad de Humanidades y Ciencias de la Educación.

Departamento de Bibliotecología

\title{
Gestión documental sonora: el ciclo de vida digital en los archivos sonoros
}

\section{Sound documental managment: the digital life cycle in the sound archives}

\section{Perla Olivia Rodríguez Reséndiz *}

* Instituto de Investigaciones Bibliotecológicas y de la Información - Universidad Nacional Autónoma de México, México | perlaolivia@gmail.com

\section{PALABRAS CLAVE}

Ciclo de vida digital

Archivos sonoros

Preservación digital sonora

Procesos documentales

\section{KEYWORDS}

Digital life cycle

Sound archives

Digital sound preservation

Documental process

\begin{abstract}
RESUMEN
La preservación de soportes sonoros analógicos (discos, casetes, cintas de carrete abierto, entre otros), se cimienta en etapas y procesos documentales que se llevan a cabo desde que se incorpora el material en el archivo hasta que es consultado. Los procesos documentales pueden ordenarse en etapas documentales que forman el ciclo de vida. Las etapas documentales empleadas en el archivo analógico quedaron en desuso ante el crecimiento de colecciones digitales. Frente a tal situación, resulta relevante revisar los procesos y determinar las etapas a través de las cuales transita un documento digital sonoro durante el ciclo de vida digital. El artículo que se presenta tiene como objetivos identificar y exponer los principales procesos que forman parte del ciclo de vida digital en la preservación digital de archivos sonoros. Para ello, se analiza literatura científica relacionada con los procesos documentales y el ciclo de vida de los archivos sonoros; además, se examinan algunos de los más relevantes y recientes planes de preservación digital de archivos sonoros formulados en bibliotecas y archivos de alcance nacional. Con ello, se exponen conceptos que definen las etapas y los procesos documentales que se llevan a cabo en un archivo analógico y se presentan los modelos de ciclo de vida digital que incluyen materiales sonoros. Como resultado se expone la identificación y descripción de los principales procesos señalados en los modelos de ciclo de vida digital; además, se propone una ordenación de las principales etapas del ciclo de vida en la preservación digital.
\end{abstract}

\section{ABSTRACT}

The analog sound preservation of media (discs, cassettes, tapes, etc) is based on documental stages and processes, from the material is enter into the archive until it is consulted. The documental processes can be arranged in stages as part of the life cycle. Analog stages became obsolete before the growth of digital collections. Therefore it is necessary to review the processes and determinate the stages involved in the digital life cycle. The object of this article is to identify and expose the main documental processes as part of the digital life cycle in the digital preservation of sound materials. The article analyze scientific literature of documentary processes and the life cycle of sound archives; in addition, it examines some of the most relevant and recent digital preservation plans for sound archives formulated by national libraries and archives. With this, the article expose the concepts to define the stages and the documentary processes that are carried in an analog archive and also it presents digital life cycle models in which sound materials are included. As a result, it will be exposed the identification and description of the main processes of the digital life cycle. In addition, it will propose an ordering of the main stages of the life cycle in the digital preservation.

Recibido: 31 de mayo de 2017 | Aceptado: 8 de septiembre de 2017 | Publicado: 9 de octubre de 2017 


\section{Introducción}

Aun cuando el interés en torno a la preservación digital surgió en la década de los años 70 del siglo XX, y se intensificó en el XXI (Baggio y Flores, 2013), las investigaciones en relación con la preservación digital y el tránsito de los documentos en un archivo digital sonoro son escasas. Esta situación obedece probablemente a que el campo de la preservación digital, como ha señalado Térmens (2009), emergió como línea de investigación hace dos décadas y por ello no está totalmente definido.

En el ámbito de los archivos sonoros, la digitalización de colecciones sonoras comenzó hace más de tres décadas (Haefner, 2002) y la prioridad, ante el inminente riesgo de pérdida, fue transferir la mayor cantidad de documentos en el menor tiempo posible. Desde entonces, se han puesto en marcha Sistemas de Gestión y Almacenamiento Masivo Digital (SGAMD) para almacenar y gestionar los contenidos (Bradley, Lei y Blackall, 2007) provenientes de la digitalización. La operación y funcionamiento de los SGAMD definieron las bases para la creación de archivos digitales, que preservan colecciones sonoras que crecen de forma exponencial y constante.

En relación con la preservación digital de archivos sonoros, se han comenzado a adoptar estándares, normas y recomendaciones internacionales creadas para guiar y normar el trabajo en archivos digitales. Para el diseño y creación de sistemas de información de archivo abierto se produjo el Open Archival Information System (OAIS). Para la concepción, gestión y conservacion de los metadatos se han formulado el Preservation Metadata Implementation Strategis (PREMIS Editorial Committe, 2015) y el Metadata Encoding and Transmission Standard (METS, 2010). Para la auditoría y certificación de repositorios digitales se ha establecido el Trusted Repositories Audit and Certification: Criteria and Checklist (OCLC, 2007); entre otros.

También se han formulado propuestas sustentables para procurar la continuidad de los trabajos en un archivo, asegurar la permanencia de los recursos económicos, garantizar la actualización de la plantilla profesional, enfrentar presiones políticas, entre otras (Rodríguez Reséndiz, 2016). Sin embargo, aún son incipientes las iniciativas para identificar las etapas y los procesos a través de los cuales transita un documento digital sonoro dentro de un archivo. Conviene señalar que las publicaciones que abordan el ciclo de vida digital de los archivos sonoros son escasas. Situación a la que se añade que en el gremio de los archivistas sonoros no existe consenso en torno a cómo preservar en plataformas digitales los archivos sonoros.

\section{Objetivos, metodología y estructura}

Este artículo tiene como objetivos identificar y analizar los principales procesos documentales que forman parte del ciclo de vida digital en la preservación digital de archivos. El método de investigación empleado en este artículo se basó en la revisión de bibliografía, artículos científicos e informes que examinan los procesos documentales que se aplican en archivos sonoros que preservan colecciones analógicas. Se recuperaron, con especial interés, las recomendaciones internacionales en la materia. Con ello, se estableció el marco de referencia de las transformaciones de los procesos documentales en un 
archivo sonoro. Después, se establecieron los procesos documentales, físicos e intelectuales.

Más adelante, se analizaron modelos de ciclo de vida digital para colecciones científicas y archivos. Se seleccionaron y estudiaron aquellos que se ocupan de materiales sonoros. Después, se describieron los modelos seleccionados y se compararon con los procesos de archivos analógicos. Para concluir, se relacionaron y establecieron los principales procesos documentales que intervienen en el ciclo de vida digital de un archivo sonoro. Asimismo, se propuso la ordenación de los procesos documentales en las principales etapas del ciclo de vida digital.

El artículo expone cómo se han modificado los procesos documentales en los archivos sonoros, a través de los diferentes períodos por los que han transitado; después explica qué se entiende por ciclo de vida digital; más adelante se presentan los modelos de ciclo de vida digital que se han propuesto y de éstos cuáles han sido diseñados para archivos sonoros; finalmente se formulan resultados y conclusiones.

\section{Discusión}

\section{La transformación de los procesos documentales en archivos sonoros}

La preservación de grabaciones de voces, música, programas de radio, paisaje sonoro y arte sonoro, entre otras manifestaciones y expresiones sonoras, es una tarea relativamente reciente. Inició después de que la UNESCO (1980) reconoció el valor patrimonial y documental de los documentos audiovisuales, en los cuales se inscriben los sonoros, y alertó de su riesgo de pérdida si no se emprendían tareas de preservación.

Los archivos sonoros tienen la misión de preservar la herencia audible. Para ello, la gestión documental se desarrolla a través de una serie de procesos documentales, desde su acopio hasta su acceso. La gestión documental se ha modificado porque también el archivo sonoro se ha transformado.

Se pueden distinguir tres períodos a través de los cuales ha transitado el archivo: la preservación de colecciones sonoras en formatos analógicos; la incorporación de nuevos procesos derivados de la digitalización y la preservación digital de colecciones sonoras.

Los primeros intentos por definir el trabajo documental en una fonoteca que preserva colecciones analógicas, se basaron en el tratamiento documental que se lleva a cabo en las bibliotecas (Miranda, 1990). Las fases de la cadena documental fueron: selección, adquisición, verificación, registro, signatura, sellado, tejuelado y catalogación.

Más adelante, Caldera Serrano (2004) planteó, desde la perspectiva del trabajo documental en los archivos radiofónicos, que el tratamiento documental de la información sonora estaba formado por la audición completa del documento, el análisis formal, el análisis de contenido, la descripción, la confección y la validación. Estas propuestas pioneras se centraron en el tratamiento documental, es decir, en la escucha y análisis del documento para su catalogación.

Con el paso del tiempo, en los archivos sonoros, se pusieron en marcha procesos específicos de acuerdo con la naturaleza sonora de este tipo de documentos. La 
conceptualización de los procesos documentales, tanto en los archivos sonoros como en los audiovisuales, devino de las actividades laborales, etapas y procedimientos de trabajo, diferenciados o simultáneos (Ojeda, 2008).

La conservación y el acceso fueron reconocidos como tareas indispensables en los archivos. La catalogación carecía de sentido si no se conservaban y se daba acceso a los contenidos. Bajo esta perspectiva, la preservación se erigió como el conjunto de tareas y procesos documentales necesarios para conservar y dar acceso (Edmondson, 2016) a los contenidos grabados en documentos sonoros. Conviene señalar que los procesos documentales son acciones específicas, intelectuales o físicas, que se llevan a cabo en un archivo sonoro. Las etapas son un conjunto de procesos documentales.

La Asociación Internacional de Archivos Sonoros y Audiovisuales (Comité Técnico IASA, 2005) identificó cuatro etapas o tareas básicas que se llevan a cabo en un archivo de fondos y colecciones analógicas: acopio, conservación, documentación y acceso. Estas etapas corresponden a una serie de procesos documentales a través de los cuales se lleva a cabo la preservación de colecciones analógicas. Los procesos documentales se basan en dos tipos de actividades: físicas e intelectuales. El acopio es una tarea intelectual, asentada en la investigación, a través de la cual se identifican colecciones susceptibles de ser incorporadas en el archivo. Una vez que las colecciones son identificadas, se trasladan al archivo, y para ello, se llevan a cabo actividades físicas (Rodríguez Reséndiz, 2012). La conservación involucra actividades físicas para la limpieza y estabilización de los soportes (Salazar, 2015). Las técnicas y tecnologías utilizadas en este proceso se aplican en función del soporte de que se trate, de acuerdo con las normas y recomendaciones internacionales establecidas en la materia (AES, 2003, 2006 y 2010; Comité Técnico IASA, 2011 y 2015; COTENNDOC, 2013). La conservación también es un proceso intelectual porque, a través de éste, se diseñan los planes de conservación preventiva y correctiva de los documentos del archivo. La documentación incluye la organización física de los soportes sonoros en las bóvedas y la catalogación es considerada una actividad eminentemente intelectual, a través de la cual se extraen y describen, de acuerdo con reglas y normas establecidas, datos del documento para su control, registro y recuperación (COTENNDOC, 2009; IASA, 2005). El acceso a los materiales analógicos es una actividad física, que durante muchos años se basó en facilitar a los usuarios el soporte y el equipo tecnológico para reproducir y escuchar los documentos (Rodríguez Reséndiz, 2012). La gestión documental que se lleva a cabo en un archivo sonoro está asociada a una serie lineal de procesos documentales, que tiene un inicio y una conclusión. La concatenación de procesos determina el flujo y roles que se desarrollan en un archivo sonoro.

La digitalización hizo posible la reproducción fidedigna del documento, modificó la perspectiva de trabajo lineal en el archivo e incorporó tareas, procesos, flujos de trabajo y roles (Rodríguez Reséndiz, 2016). A través de este proceso se transfirieron contenidos grabados en diversos soportes analógicos a plataformas digitales: como son obsoletos, ya no existe equipo para su reproducción y el mantenimiento técnico ha desaparecido (Comité Técnico IASA, 2005). Además, determinó la intersección entre los procesos documentales de colecciones analógicas y la preservación digital como método de preservación de contenidos. Con ello, se definió la transición del archivo analógico al digital. 
La diferencia entre la preservación de materiales analógicos y digitales radica en que, mientras los primeros pueden permanecer almacenados, en reposo durante varios años o décadas, los objetos digitales, una vez que se incorporan en plataformas digitales, deben tener una gestión continua (Besser, Cariani y Van Malssen, 2010; Ng, Rubin y Van Malsen, 2010) a través de procesos y métodos de preservación digital.

A través de la preservación digital se conservan, se da acceso y difunden, a largo plazo, los contenidos digitales sonoros. El documento o ítem digital transita a través de diferentes etapas y procesos desde el momento en que fue creado hasta que es consultado o reutilizado. Estas etapas forman parte del ciclo de vida digital.

En la preservación digital los procesos documentales son intelectuales, es decir, no se llevan a cabo actividades que implican destrezas físicas. Estos procesos se desarrollan a través del uso de tecnología informática. Así, labores relacionadas con la manipulación para la limpieza, estabilización y organización, entre otras, han sido sustituidas por procesos intelectuales. Un ejemplo de ello es la verificación de la autenticidad e integridad de los documentos, proceso que no requiere de la manipulación física del documento.

\section{El ciclo de vida digital}

El término ciclo de vida

es un modelo o concepto clásico de la archivística moderna. Se refiere a las distintas fases o etapas por las que pasan los documentos y a las transformaciones en sus valores legales, informativos, etcétera, desde su creación como coproductos de la actividad humana hasta su eliminación o selección para su custodia permanente, generalmente por su valor histórico (Voutssás, 2010, p. 158).

De forma análoga, el ciclo de vida digital evidencia las etapas, procesos y modificaciones por los que atraviesan los materiales digitales, desde su creación hasta su uso o reaprovechamiento documental. Incide en la toma de decisiones e intervención en un archivo, a largo plazo y de forma continua, y determina los roles dentro de un archivo que preserva colecciones digitales.

El ciclo de vida digital establece el marco de referencia que describe la secuencia de acciones o fases para la gestión de objetos digitales (Waddington et al., 2016). El término ciclo de vida fue incorporado en el modelo OAIS (Wilson et al., 2006) y es utilizado tanto en archivos que resguardan materiales analógicos como digitales.

Para explicar el ciclo de vida digital se han formulado modelos que son creaciones abstractas que ayudan a comprender la secuencia de etapas o tareas que se desarrollan durante la preservación digital y que "pueden usarse en la planeación organizacional a nivel superior y en la detección de deficiencias en los procedimientos" (Waddington et al., 2016, p. 54) en el archivo.

\section{Los modelos de ciclo de vida}

Los primeros intentos por explicar el flujo por el que transitan los objetos digitales datan de la década de 1990, cuando Upward (1996) formuló el modelo de registro continuo. Este 
modelo es una propuesta abstracta para comprender y explorar las actividades de preservación de los registros de un archivo; esto bajo la consideración de que el contenido y la estructura del registro son fijos, pero el contexto puede cambiar en el tiempo. Por lo tanto, el registro siempre está en el devenir, tal como advierte el autor. Con ello, se advirtió que "la gestión del documento es un proceso continuo desde el momento de su creación" (Rodríguez Bravo, 2007, p. 362).

Tabla 1. Modelos del ciclo de vida digital

\begin{tabular}{|l|}
\hline $\begin{array}{l}\text { I2S2 Idealized Scientific Research Activity Lifecycle } \\
\text { Model }\end{array}$ \\
\hline DDI Combined Life Cycle Model Data \\
\hline ANDS Data Sharing Verbs \\
\hline DataONE Data Lifecycle \\
\hline UK Data Archive Data Lifecycle \\
\hline Research360 Institutional Research Lifecycle \\
\hline $\begin{array}{l}\text { Capability Maturity Model for Scientific Data } \\
\text { Management Crowston }\end{array}$ \\
\hline DCC Curation LifecycleModel \\
\hline Research data strategy working \\
\hline Paradigm \\
Personal Archives Accesible in Digital Media \\
\hline Interpares \\
\hline Life-cycle model for moving images and sound \\
\hline Nederlands Instituut voor Beeld en Geluid \\
\hline
\end{tabular}

Fuente: Elaboración propia con información de Ball (2012).

Como se puede observar en la Tabla 1, durante los primeros años del siglo XXI se han formulado una amplia gama de modelos de ciclo de vida. Cada modelo expresa procesos y etapas a través de las cuales transitan los ítems u objetos digitales en un repositorio o archivo digital. La mayoría de las propuestas se han centrado en explicar el ciclo de vida digital en la producción científica; en menor proporción se han creado las destinadas a exponer los procesos por los que atraviesa un objeto en un archivo digital.

Se carece de un modelo único de ciclo de vida digital creado exclusivamente para archivos sonoros. En tres modelos, el ciclo de vida digital fue diseñado para incorporar documentos sonoros, junto con otros tipos de documentos como son video, film, fotografías, sitios web, y videojuegos.

Los modelos que consideran a los documentos sonoros son: el Life-cycle model for moving images and sound (Wilson et al., 2006), el Curation Lifecycle Model del Digital Curation Centre (DCC) de Reino Unido y el modelo propuesto por el Nederlands Instituut voor Beeld en Geluid (De Jong, 2016), institución pública de Holanda.

Los modelos enunciados se basan y/o complementan con el uso de modelos y estándares internacionales creados para la gestión y administración de colecciones digitales. Los tres modelos se sustentan en el OAIS. El Life-cycle model for moving images and sound (Wilson et al., 2006) coincide con el TDR (Trusted Digital Repositories), que brinda una 
serie de requerimientos de fiabilidad y confianza en un repositorio. En tanto que el modelo propuesto por el DCC se complementa con el ISO15489 (Higgins, 2008).

El Life-cycle model for moving images and sound, formulado por Wilson, et al. (2006) identifica eventos, acciones, procesos y políticas que sustentan la preservación digital, tanto para documentos sonoros como audiovisuales. Con ello, se pretende construir propuestas organizativas y planificar la preservación de los objetos digitales a largo plazo, a través de métodos de conservación activos e intervencionistas. De acuerdo con este modelo, la preservación digital se desarrolla en seis eventos o sucesos: creación, ingesta o transferencia, curaduría y preservación, acceso y uso, acciones de monitoreo de software y hadware, así como rechazo o retirada de materiales.

La creación está determinada por las decisiones tomadas en el momento de producir el material sonoro o audiovisual. Involucra los formatos en que serán grabados los materiales, los metadatos de origen, la calidad en que son producidos los documentos y los derechos de autor. Vincula al usuario con el creador, considerando costos, recursos, estrategias de selección y derechos de autor. La creación es, de acuerdo con los autores, un proceso fundamental para la preservación sustentable. La ingesta o transferencia, está formada por un conjunto de acciones a través de las cuales se asegura la preservación de los materiales a largo plazo. Las acciones son: verificar el formato; revisar que los materiales están libres de virus antes de ser incorporados al archivo; realizar el proceso de cheksum o comprobación de la integridad de los objetos digitales, para confirmar que están completos y no están dañados; preparar la documentación y los metadatos y comprobar la legibilidad para que los materiales permanezcan accesibles y usables en el tiempo. Una vez que el material ha sido transferido al archivo, inicia la curaduría y preservación. Se debe normalizar el formato que se preservará y decidir cuántas copias se crearán y conservarán; se asignarán los identificadores persistentes y se verificará la integridad. Se definirá, en función de los derechos, el acceso futuro y las posibilidades de uso del material. Otro evento es el acceso y uso de las colecciones que son consultadas por parte de los usuarios. Por lo tanto, se deben realizar acciones de monitoreo de software y hadware para llevar a cabo procesos de migración o emulación de colecciones, a partir de políticas y procedimientos establecidos en el archivo. El rechazo o retirada de materiales forma parte del ciclo de vida; esta decisión debe basarse en políticas y procedimientos claramente establecidos. "Cuando se retiran los recursos audiovisuales, éstos pueden ser eliminados físicamente del repositorio, pero la mejor práctica seguida por muchos repositorios es mantener los recursos originales, pero marcarlos como no disponibles o reemplazados por una versión más reciente" (Wilson et al., 2006, p. 99).

La primera versión del Curation Lifecycle Model del DCC fue publicada en 2007 (Higgins, 2008), y después de un año de consultas se concluyó. Este modelo inglés, identifica y relaciona acciones, roles y responsabilidades. Además, permite construir el marco de referencia para el uso de estándares y tecnologías necesarias para la preservación digital. Puede ser usado para asegurar que los procesos y políticas de preservación estén debidamente documentados (DCC, 2017). El DCC, como es conocido este modelo, puede ser aplicado tanto en repositorios científicos como en archivos digitales que resguardan grabaciones electrónicas (Harvey, 2010). El centro del modelo son los datos, que pueden ser objetos digitales o bases de datos (DCC, 2017). Las bases de datos son colecciones 
estructuradas de archivos o datos almacenados en un sistema de cómputo. Los objetos digitales pueden ser simples o complejos. Los simples son ítems discretos, como archivos de texto, sonidos o imágenes, y metadatos asociados. Los complejos, son objetos discretos, producidos con la combinación de otros objetos, por ejemplo, las páginas web (DCC, 2017). El modelo está formado por tres grupos de acciones: ciclo de vida completo, secuenciales y ocasionales.

Las acciones del ciclo de vida son: descripción y representación de la información a través de la asignación de metadatos administrativos, descriptivos, técnicos y estructurales para asegurar la preservación a largo plazo; plan de preservación, que incluye gestión y administración, durante todo el ciclo de vida digital; observación y participación de la comunidad para mantener un control de las actividades e intervenir en el desarrollo de estándares, herramientas y software; así como cuidado y preservación, a través de promover acciones para la conservación y acceso de los contenidos (DCC, 2017). Las acciones secuenciales necesarias para el cuidado de los datos se desarrollan a través de todo el ciclo de vida, desde la creación hasta el uso y reuso (Harvey, 2010). Las acciones secuenciales son: conceptualización; creación y recepción; evaluación y selección; ingesta; acciones de conservación; almacenamiento; acceso, uso y reuso; y transformación. La transformación no se lleva a cabo fuera del ciclo de vida, se repite durante el cuidado de los datos. Lo que significa que la transformación de los datos, a través del proceso de reuso, puede generar un nuevo set de datos. Las acciones ocasionales suceden cuando se presentan condiciones específicas, pero no se aplican a todos los datos. Las acciones ocasionales son: disposición o transferencia de datos que no han sido seleccionados para su cuidado y preservación digital a largo plazo; reevaluación de datos que fallan y migración de datos contenidos en diferentes formatos (Higgins, 2008). El DCC incorpora, además del término preservación, el de curaduría para explicar el ciclo de vida digital. La curaduría digital se refiere a mantener y agregar valor a la información digital para su acceso actual y futuro. Es la gestión activa en el ciclo de vida digital (Pennock, 2007).

En el Nederlands Instituut voor Beeld en Geluid, el modelo de ciclo de vida está determinado por el flujo de trabajo del archivo digital, que consta de tres procesos: ingesta, almacenamiento y acceso. Este modelo registra las acciones que puede tener un objeto digital y define la composición (cambiante) del objeto durante todos los procesos. Las actividades relacionadas con los metadatos también se registran. Cada una de las actividades realizadas están controladas, son verificables y corresponden a la política de preservación del archivo (De Jong, 2016).

Los objetos digitales son paquetes de información que cambian desde el momento mismo en que son incorporados al archivo, hasta que son consultados. Previo a la ingesta de los materiales, se negocia el depósito de los materiales y se establecen con precisión los formatos, derechos de autor y metadatos. En el proceso de ingesta se lleva a cabo el fixity check o verificación del archivo, para identificar si los contenidos originales fueron transferidos de forma correcta. Después se determinan los formatos y metadatos técnicos. Los paquetes de información se identifican con un número, único e irrepetible. Con ello, se concluye la fase de ingesta y de incorporación del paquete de información que está listo para el almacenamiento. De acuerdo con el OAIS, esta etapa corresponde al Submission 
Information Package (SIP). Este paquete de información, en el que se incluyen los metadatos técnicos y la información del contexto de la producción, se convierte en AIP (Archival Information Package), paquete de información que se ingresa al archivo digital. Esta es la etapa de almacenamiento digital. La última etapa del ciclo de preservación digital es el DIP (Dissemination Information Package), que inicia con la identificación de un usuario registrado en el sistema que solicita un determinado tipo de documento para un uso específico. Si el sistema aprueba la solicitud, se entrega el documento, de acuerdo con el formato de acceso establecido (De Jong, 2016). Durante las etapas del ciclo de vida digital se generan de forma automática datos administrativos y técnicos, del estado de conservación de las colecciones, con el propósito de procurar la integridad y autenticidad de los datos.

Tabla 2. Ciclo de vida digital en un archivo sonoro

\begin{tabular}{|c|c|c|c|c|c|c|}
\hline \multicolumn{2}{|c|}{$\begin{array}{c}\text { Etapas del ciclo de vida digital } \\
\text { de un archivo sonoro }\end{array}$} & $\begin{array}{l}\text { Creación de } \\
\text { documentos }\end{array}$ & $\begin{array}{l}\text { Ingesta y } \\
\text { evaluación }\end{array}$ & $\begin{array}{l}\text { Curaduría, conservación y } \\
\text { almacenamiento }\end{array}$ & \multicolumn{2}{|c|}{ Acceso, uso y reuso } \\
\hline & & & & \multicolumn{3}{|c|}{ Retirada o rechazo } \\
\hline $\begin{array}{l}\text { Life-cycle model } \\
\text { for moving images } \\
\text { and sound }\end{array}$ & & Creación & $\begin{array}{l}\text { Ingesta } 0 \\
\text { transferencia }\end{array}$ & Curaduría y preservación & Accesoy uso & $\begin{array}{c}\text { Acciones de } \\
\text { monitoreo de } \\
\text { softwarey } \\
\text { hadware }\end{array}$ \\
\hline \multirow{4}{*}{$\begin{array}{l}\text { Curation Lifecycle } \\
\text { Model del DCC }\end{array}$} & \begin{tabular}{|l} 
Descripción y \\
representación de \\
la información \\
\end{tabular} & \multirow{4}{*}{$\begin{array}{l}\text { Conceptua lización; } \\
\text { Creación y recepción; }\end{array}$} & \multirow{4}{*}{$\begin{array}{c}\text { Evaluacióny } \\
\text { selección; ingesta; }\end{array}$} & \multirow{4}{*}{$\begin{array}{l}\text { Acciones de conservación; } \\
\text { almacenamiento; }\end{array}$} & \multirow{4}{*}{$\begin{array}{c}\text { Acceso, uso, reuso y } \\
\text { transformación }\end{array}$} & \\
\hline & $\begin{array}{c}\text { Plande } \\
\text { preservación }\end{array}$ & & & & & \\
\hline & $\begin{array}{c}\text { Observación y } \\
\text { participación de } \\
\text { la comunidad }\end{array}$ & & & & & \\
\hline & \begin{tabular}{|c|c|}
$\begin{array}{c}\text { Cuidadoy } \\
\text { preservación }\end{array}$ \\
\end{tabular} & & & & & \\
\hline $\begin{array}{l}\text { Nederlands } \\
\text { Instituut voor } \\
\text { Beeld en Geluid }\end{array}$ & & & Ingesta & Al macenamiento & Acceso & \\
\hline
\end{tabular}

Fuente: Elaboración propia con información de Wilson et al., 2006; DCC, 2017 y De Jong, 2016.

\section{Resultados}

1. La preservación digital debe basarse en la gestión documental de las etapas y los procesos que forman parte del ciclo de vida del documento sonoro digital.

2. El ciclo de vida digital está formado por un conjunto de etapas a través de las cuales transita el objeto digital (media y metadata) desde que es incorporado al archivo hasta que es consultado y reutilizado o reaprovechado. Cada etapa está formada por una secuencia de procesos intelectuales que se llevan a cabo por medio de equipos informáticos. 
3. El ciclo de vida de las colecciones analógicas está formado por las etapas de acopio, documentación, conservación y acceso. A su vez, cada etapa se compone de procesos documentales que pueden ser intelectuales y físicos.

4. La preservación digital también se integra por una serie de etapas. Cada etapa está constituida por una secuencia de procesos intelectuales que se caracterizan por el uso de tecnología.

5. El ciclo de vida digital, a diferencia de los procesos documentales analógicos que se desarrollan de forma lineal, se repite de forma regular y continua cada cierto tiempo. Así, la gestión documental digital es una tarea permanente que nunca concluye.

6. El ordenamiento de los procesos documentales, de acuerdo con el análisis de los modelos de ciclo de vida antes expuesto (como se señala en la Tabla 2), propone las siguientes etapas que forman el ciclo de vida digital:
a) creación de documentos
b) ingesta y evaluación
c) curaduría, conservación y almacenamiento
d) acceso, uso y reuso.

7. Los modelos de ciclos de vida digital estudiados, a diferencia de los procesos documentales analógicos, incorporan a la creación como un proceso previo al acopio de los materiales en el archivo. Éste corresponde a la etapa de producción del documento sonoro en diversos formatos. En el ciclo de vida digital la creación se puede normar a través de la puesta en marcha de lineamientos, en instituciones productoras de documentos sonoros como las radiodifusoras, o bien en instituciones públicas o privadas, cuya misión es crear contenidos digitales sonoros. Sin embargo, en los archivos sonoros y bibliotecas se reciben materiales en una amplia gama de formatos y no se puede normar su creación. Por lo tanto, esta etapa generalmente queda fuera del control del archivo.

8. La ingesta y evaluación ocupan un rol destacado en el ciclo de vida digital. Se evalúan los formatos, el contenido, los metadatos de origen y los derechos de autor de los materiales. En los modelos estudiados se han incorporado la revisión y comprobación de contenidos como parte del ciclo de vida digital. En éste proceso se verifica la integridad de los materiales digitales. Asimismo, se consideran la migración y el monitoreo de software y hadware como tareas periódicas a realizar en un archivo digital.

9. La curaduría, la conservación y el almacenamiento aparecen como tareas asociadas. En los archivos analógicos, la conservación se basa en mantener los documentos sonoros en condiciones de temperatura y humedad en las bóvedas; mientras que, en el ámbito digital, la conservación se ocupa de garantizar la permanencia del almacenamiento digital. Asimismo, aparece la curaduría como la 
tarea intelectual por medio de la cual se decidirá cuántas copias se crearán y conservarán para su uso y reaprovechamiento futuro. La curaduría como proceso agrega valor al contenido.

10. El acceso es la última etapa en la preservación de colecciones analógicas. En la preservación digital, el uso, reuso y reaprovechamiento es la etapa a partir de la cual un paquete de información se transforma en un nuevo set de datos.

\section{Conclusiones}

La gestión documental se ha transformado a través de tres periodos por los que ha transitado el archivo sonoro: la preservación de colecciones sonoras en formatos analógicos, la incorporación de los nuevos procesos derivados de la digitalización y la preservación digital de colecciones sonoras. Estos cambios se pueden evidenciar en las etapas y los procesos documentales.

La preservación de colecciones analógicas se desarrolla en cuatro etapas (acopio, documentación, conservación y acceso). Cada etapa integra procesos documentales que pueden ser físicos o intelectuales. La digitalización incorporó tareas, flujos, roles y modificó los procesos documentales. Fue el punto de partida para la transición del archivo analógico al digital. El documento sonoro digital, al igual que el analógico, recorre una serie de etapas y procesos documentales que conforman el ciclo de vida.

Desde hace más de un cuarto de siglo se ha formulado una amplia gama de modelos de ciclo de vida digital. De éstos, se identifican tres que incluyen a los documentos sonoros: Life-cycle model for moving images and sound, el Curation Lifecycle Model del Digital Curation Centre (DCC) de Reino Unido, y el modelo propuesto por el Nederlands Instituut voor Beeld en Geluid. Cada modelo explica de forma diferente el tránsito de los objetos digitales sonoros. A partir del análisis de éstos se propone que las principales etapas del ciclo de vida digital de un archivo sonoro son:
a) creación de documentos
b) ingesta y evaluación
c) curaduría, conservación y almacenamiento
d) acceso, uso y reuso.

El ciclo de vida digital se basa en la gestión de objetos digitales durante la preservación digital del archivo sonoro. Comprender las principales etapas por las cuales transitan los documentos digitales contribuye a tener una idea de la operación del archivo digital, a través del flujo de los paquetes de información, los roles que se asumen y las políticas necesarias para su preservación a largo plazo. 


\section{Referencias bibliográficas}

AES. (2003). Standard for audio preservation and restauration -Storage and handlingStorage of polyester- base magnetic tape (ISO 18923:2000).

AES. (2006). Information document for preservation of audio recordings-Extended term storage enviroment for multiple media archives (ISO18934:2011).

AES. (2010). Standard for audio preservation and restoration -Magnetic tape- Care and handling practices for extended usage (ISO 18933:2012).

Baggio, C., y Flores, D. (2013). Documentos digitais: preservação e estratégia. Biblos: revista do Instituto de Ciências Humanas e da Informação, 27(1). Recuperado de https://www.seer.furg.br/biblos/article/view/2654/2395

Ball, A. (2012). Review of data management lifecycle models. Bath: University of Bath. Recuperado de http://opus.bath.ac.uk/28587/

Besser, H., Cariani, K., y Van Malssen, K. (2010). Six strategies for sustainable preservation of born digital public television. Archiving 2010 - Preservation Strategies and Imaging Technologies for Cultural Heritage Institutions and Memory Organizations. Washington: Digital Information Infraestructure and Preservation Program (NDIIPP) of the Library Congress. Recuperado de http://www.thirteen.org/ptvdigitalarchive/files/2009/10/PDPTV SustainabilityStrategies.p df

Bradley, K., Lei J., y Blackall, Ch. (2007). Towards and open source repository and preservation system: recommendations on the implementation of an open source digital archival and preservation system on related software development. Paris: UNESCO. Recuperado de http://old.nlai.ir/Portals/6/pdf/Towards\%20an\%200pen\%20Source \%20Archival\%20Repository\%20and\%20Preservation\%20.pdf

Caldera Serrano, J. (2004). La documentación sonora en los sistemas de información documental de los medios audiovisuales. Boletín de la Asociación Andaluza de Bibliotecarios, 74, 29-39. Recuperado de http://eprints.rclis.org/6203/

Comité Técnico IASA. (2005). TC-03 La salvaguarda del patrimonio sonoro: Ética, principios y estrategia de preservación. México: IASA- Radio Educación.

Comité Técnico IASA. (2011). TC-04 Directrices para la producción y preservación de objetos digitales de audio. Comité Técnico de Estándares, Prácticas y Estrategias Recomendadas. Madrid: AEDOM-IASA.

Comité Técnico IASA. (2015). TC-05. Manejo y almacenamiento de soportes de audio y de video. Comité Técnico Normas, prácticas recomendadas y estrategias). México: Fonoteca Nacional.

COTENNDOC. (2009). Norma Mexicana de Catalogación de Documentos Fonográficos (NMX-R-002-SCFI-2009). México: COTENNDOC. Recuperado de http://www.casae.org/docs/Norma mexicana catalogacion archivos sonoros.pdf 
COTENNDOC. (2013). Norma Mexicana de Documentos Videográficos y Fonograficos. Lineamientos para su conservación (NMX-R-053-SCFI-2013). México: CONTENNDOC.

De Jong, A. (2016). Digital preservation sound and vision. Policy, standards and procedures. The Haye: Netherlands Institute for Sound and Vision. Recuperado de http://publications.beeldengeluid.nl/pub/388

DCC. (2017). Digital Curation Centre. Edinburgh: Digital Curation Centre. Recuperado de http://www.dcc.ac.uk/

Edmondson, R. (2016). Audiovisual archiving philosophy and principles. París: UNESCO.

Haefner, A. (2002). Strategies and preservation policies in the radio archive of the German public broadcaster SWR. Cuadernos de documentación multimedia, 13. Recuperado de https://dialnet.unirioja.es/servlet/articulo?codigo $=870817$

Harvey, R. (2010). Digital curation: a how to do It manual. New York: Neal Schuman Publishers, INC.

Higgins, S. (2008). The DCC curation lifecycle model. International journal of digital curation, 3(1), 134-140. Doi: http://doi.org/10.1145/1378889.1378998

IASA. (2005). Reglas de catalogación de la IASA. Manual para la descripción de registros sonoros y documentos audiovisuales relacionados. Madrid: Asociación Internacional de Archivos Sonoros y Audiovisuales (IASA).

METS. (2010). Metadata encoding \& transmission standard. Recuperado de http://www.loc.gov/standards/mets/mets-schemadocs.html

Miranda, F. (1990). La fonoteca. Madrid: Fundación Germán Sánchez Ruipérez.

Ng, Y., Rubin, N. y Van Malsen (2010). Strategies for sustainable preservation of born digital public television. Washington: Library of Congress.

OCLC. (2007). Trustworthy repositories audit \& certification: criteria and checklist. Washington: OCLC. Recuperado de http://www.crl.edu/sites/default/files/d6/attachments/pages/trac 0.pdf

Ojeda, G. (2008). Los archivos audiovisuales en las redes digitales de comunicación para la educación y la cultura. Informe de Investigación y Documentación Analítica. Madrid: Ministerio de Educación y Ciencia de España. Recuperado de http://tecnologiaedu.us.es/nweb/htm/pdf/versionpdf.pdf

Pennock, M. (2007). Digital curation: a life cycle approach to managing and preserving usable digital information. Library and archives journal, 1 . Recuperado de http://www.ukoln.ac.uk/ukoln/staff/m.pennock/publications/docs/lib-arch_curation.pdf

PREMIS Editorial Committe (2015). PREMIS data dictionary for preservation metadata, version 3.0. Recuperado de https://www.loc.gov/standards/premis/v3/premis-3-0final.pdf

Rodríguez Bravo, B. (2007). Los repositorios de información, guardianes de la memoria. Anales de documentación, 10 Recuperado de 
http://revistas.um.es/analesdoc/article/view/1251/1301

Rodríguez Reséndiz, P. O. (2012). El archivo sonoro. Fundamentos para la creación de una fonoteca nacional. México: Editorial Library Outsourcing.

Rodríguez Reséndiz, P. O. (2016). El OAIS en la preservación digital de archivos sonoros. Investigación bibliotecológica: archivonomía, bibliotecología e información, 30(70). http://doi.org/10.1016/j.ibbai.2016.10.009.

Salazar, M. (2015). Documentos sonoros. En Romero, M. (Coord), Conservación de documentos analógicos y digitales. Madrid: Editorial Nerea.

Térmens, M. (2009). Investigación y desarrollo en preservación digital: un balance internacional. El profesional de la información, 18(6). http://doi.org/10.3145/epi.2009.nov.04

Upward, F. (1996). Structuring the Records Continuum - Part One: Post custodial principles and properties. Archives and manuscripts, 24(2).

UNESCO (1980). Recomendación sobre la salvaguarda y la conservación de las imágenes en movimiento. Recuperado de http://portal.unesco.org/es/ev.php$\underline{U R L}$ ID $=13139 \& U R L \quad D O=D O$ TOPIC\&URL SECTION=201.html

Voutssás, J. (2010). La cadena de preservación en archivos digitales. En Archivos electrónicos - textos y contextos. Recuperado de http://iibi.unam.mx/voutssasmt/documentos/preservacion digital y cadena.pdf

Waddington, S. et al. (2016). PERICLES - digital preservation through management of change in evolving ecosystems. The success of European projects using new information and communication technologies. Recuperado de http://www.scitepress.org/DigitalLibrary/PublicationsDetail.aspx?ID =rl7EWgOBdCY =\&t=1 Wilson, A. et al. (2006). Digital moving images and sound archiving. Arts and humanities data center. Recuperado de https://www.prestocentre.org/system/files/library/resource/movingpicturesandsoundarchi vingfinalversion.pdf 